\title{
State of the Art Microanalysis Using Raman Microscopy
}

\author{
Peng Wang
}

Bruker Optics Inc., Billerica, MA, USA

\section{Introduction}

Raman spectroscopy is one of the most common techniques for materials identification and characterization. Based on inelastic scattering of a monochromatic excitation source, Raman measures vibrational, rotational and other low-frequency modes in a system. It's not only characteristic for functional groups, but also sensitive to backbone and crystalline structures. Therefore, Raman technique finds extensive applications in various fields including but not limited to pharmaceuticals, polymers, forensics, cultural heritage \& art, life science, mineralogy, materials science and so on. Especially for a system that requires microanalysis, Raman spectroscopy offers further advantages such as minimum sample consumption, minimal sample preparation, sub-micron spatial resolution, confocal measurement and many more. Senterra Raman microscope is a compact system that combines numerous novel and patented features. Controlled by the user friendly OPUS software that incorporates wizard guided measurement and interactive 3D display and evaluation tools, microanalysis was made easy and fun. In this paper, some unique applications of Raman microscopy are presented and discussed.

\section{Instrumentation}

The Senterra dispersive Raman microscope, as shown in Figure 1, is built on an Olympus BX51 optical microscope base. It has full range of optical microscope tools including aperture stop, dark field illumination, polarized light observation, Nomarski DIC enhancement and fluorescence illumination to allow the user to achieve best visualization of the sample. Senterra accommodates up to 4 excitation lasers selecting from 6 different wavelengths as $488 \mathrm{~nm}, 532 \mathrm{~nm}, 633 \mathrm{~nm}, 785 \mathrm{~nm}, 830 \mathrm{~nm}$ and the $1064 \mathrm{~nm}$ FT-Raman add-on, which significantly increases research capabilities. The system performs automatic and continuous wavelength calibrations to provide wavelength accuracy of $0.1 \mathrm{~cm}^{-1} \mathrm{RMS}$. In addition, on-demand confocal or high-throughput measurement is made possible by utilizing a computer controlled array of pinholes and slits. Very importantly, Senterra is equipped with a high performance automatic stage with $0.1 \mu \mathrm{m}$ positioning accuracy which allows very precise mapping measurements.

\section{Confocal Depth Profiling Scan of Polymer Laminate}

Depth profiling scan is one of the unique applications of Raman microscopy. In this example, a depth profile was recorded with the SENTERRA Raman-microscope over a range of $68 \mu \mathrm{m}$ with a step width of $1 \mu \mathrm{m}$ on a thin multi-component polymer laminate. For optimal performance a 100x oil immersion objective was used in combination with a $785 \mathrm{~nm}$ laser. From the measurement, representative spectra were achieved for each layer. The laminate materials could then be identified using the library search feature of the OPUS software. Combing with z-axis information, a reconstructed model of the multilayer was generated and shown in Figure 2 together with corresponding Raman spectra for each layer.

\section{Polymorphism Study of Active Pharmaceutical Ingredients (API)}

Polymorphism study of API is very important in pharmaceutical industry as polymorphic form of an API may influence its kinetics of solubilization and thus affect the release of API from the finished dose of a drug. Raman spectroscopy is an efficient tool to identify different polymorphs based on their 
spectral differences. In this study, powdered API was mapped using Senterra Raman microscope. At least two polymorphs were identified based on their characteristic Raman spectra. Chemical images of the two polymorphs were generated and shown in Figure 3.

\section{Summary}

Raman microscopy has unique applications in analyzing small samples and multicomponent systems. It not only provides molecular identification of the materials but also infers many other properties such as crystalline forms, stress and so on.
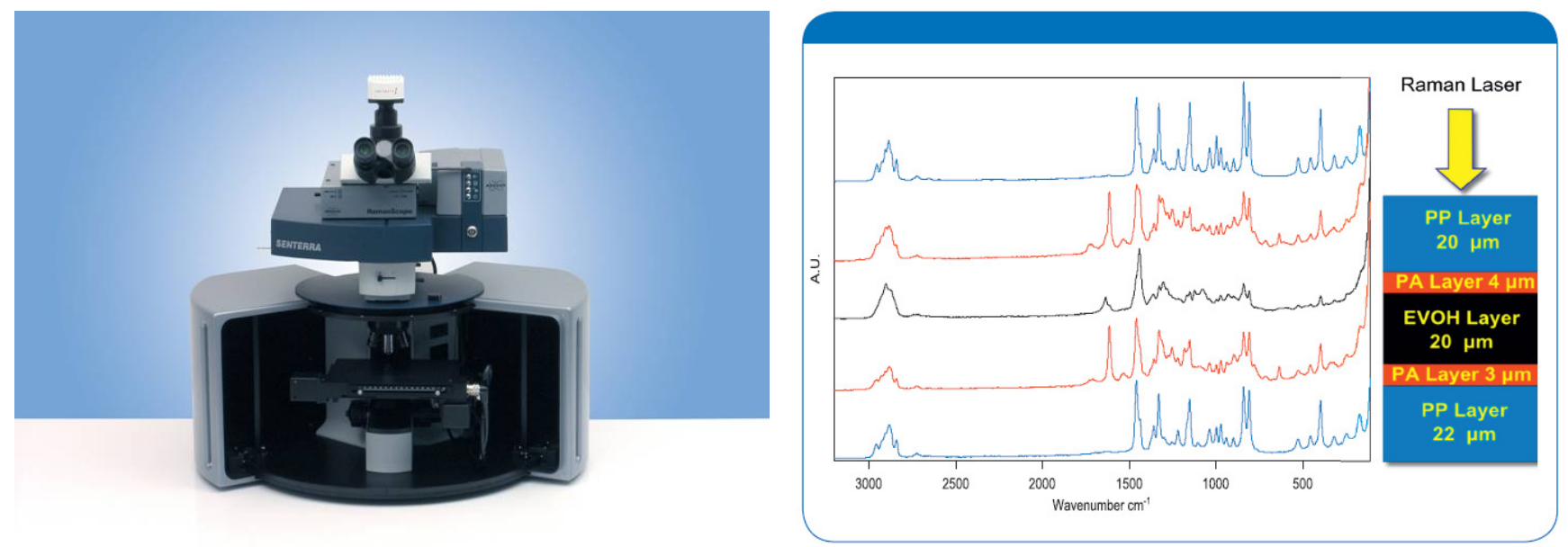

Figure 1. Senterra Raman Microscope.

Figure 2. Depth profiling scan of laminate samples.

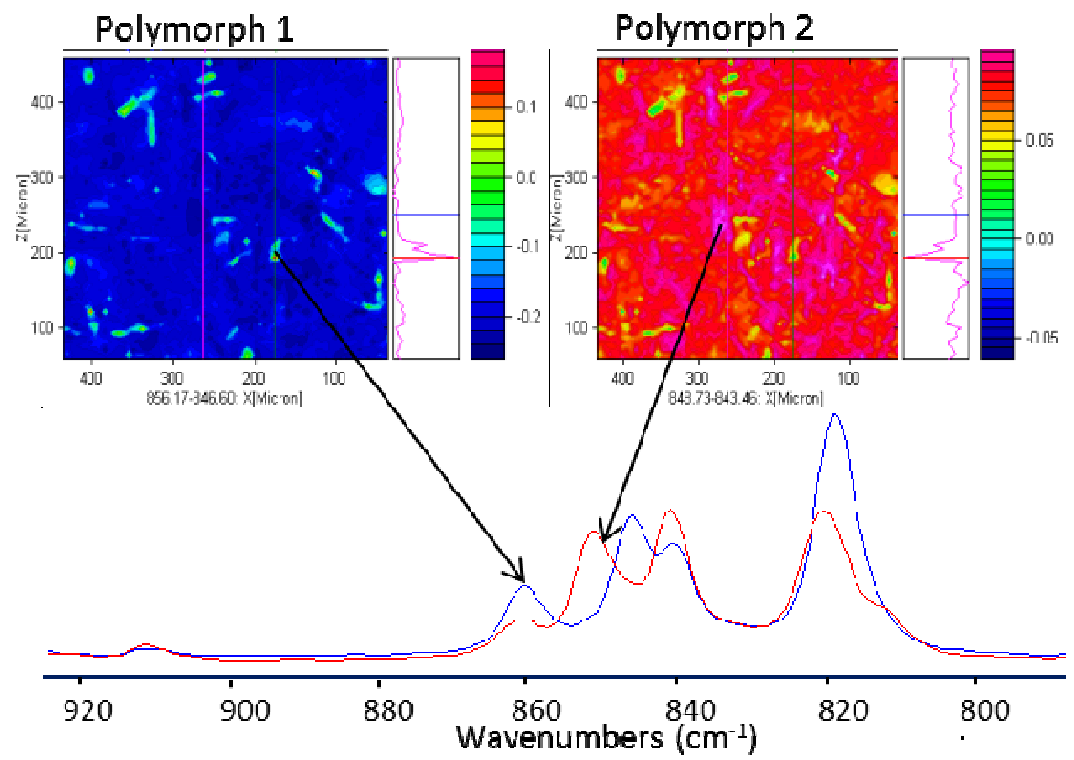

Figure 3. Two polymorphs of the API powder. 